\title{
Análisis sobre la Evaluación de la Calidad Educativa en América Latina: Caso Colombia
}

\author{
Analysis on the Evaluation of Educational Quality in Latin \\ America: Case Colombia
}

\author{
Deivis Robinson Mosquera Albornoz* \\ Universidad Católica de Oriente
}

\begin{abstract}
En el presente texto se pretende reflexionar en torno al concepto de evaluación de la calidad educativa, tratando de entender algunos de los elementos que están implícitos en él, como lo es el sentido de subjetividad y por ende la multiplicidad de interpretaciones que este posee, lo anterior teniendo en cuenta que el hecho de aplicar procesos evaluativos en el campo educativo, se encuentra rodeado de innumerables variables, donde las particularidades del contexto, deben iniciar a tener un papel relevante entorno no solo a los instrumentos que se utilizan, sino también en los resultados de las mismas. De igual forma, es relevante el poder analizar el contex to global y local, con respecto a las razones por las cuales este tipo de pruebas no están contribuyendo a la equidad y la diferenciación que debe existir entre las diferentes formas de aprender de los individuos, el pensar en estandarizar desconoce en gran medida la diversidad, las múltiples condiciones sociales, la cultura, los recursos y los contextos en los que se educan los estudiantes. A su vez, el objetivo de este escrito fue realizar un análisis sobre la situación actual de las evaluaciones estandarizadas en América Latina, tomando como base el caso colombiano, debido al aumento de la participación de los países de la región en el desarrollo de estas; es importante mencionar que lo que se busca es mostrar en primer lugar los aspectos que permitan identificar la importancia de dichas pruebas, al igual que las funciones que estas brindan a los sistemas educativos. Es de anotar que la metodología utilizada fue cualitativa, basada en un enfoque hermenéutico que utiliza como técnica fundamental el análisis documental, dando como resultado un estudio monográfico.
\end{abstract}

Palabras claves: Evaluación estandarizada; Calidad educativa; Política educativa; Medición; Pruebas internacionales; Competencias; Sistemas educativos.

In the present text it is intended to reflect on the concept of evaluation of educational quality, trying to understand some of the elements that are implicit in it, as is the sense of subjectivity and therefore the multiplicity of interpretations that it has, previous taking into account that the fact of applying evaluative processes in the educational field, is surrounded by innumerable variables, where the particularities of the context, must begin to have a relevant role not only to the instruments that are used, but also in the results of the same. Similarly, it is relevant to be able to analyze the global and local context, with respect to the reasons why this type of evidence is not contributing to equity and the differentiation that must exist between the different ways of learning of individuals, the thinking about standardizing largely ignores the diversity, the multiple social conditions, the culture, the resources and the contexts in which students are educated. In turn, the objective of this paper was to analyze the current situation of standardized evaluations in Latin America, based on the Colombian case, due to the increased participation of the countries of the region in the development of these; It is important to mention that what is sought is to show in the first place the aspects that allow to identify the importance of said tests, as well as the functions that these provide to the educational systems. It should be noted that the methodology used was qualitative, based on a hermeneutical approach that used documentary analysis as a fundamental technique, resulting in a monographic study.

Keywords: Standardized evaluation; Educational quality; Educational policy; Measurement; International tests; Competences; Educational systems.

\footnotetext{
*Contacto: deromoal@gmail.com 


\section{Introducción}

El presente artículo, pretende mostrar elementos que permitan entender un poco sobre el origen del concepto de evaluación de la calidad educativa, entendiendo que este migró del sector económico, donde se buscaba mejorar no solo los niveles de producción, sino también la calidad de los productos que se fabricaban. Además, se buscará mostrar la evolución que ha tenido dicho concepto a lo largo de la historia, con el fin de poder entender los avances o retrocesos que ha podido tener en el contexto educativo.

Asimismo, se pondrán en evidencia tanto los posibles riesgos que pueden estar implícitos en la realización de dichas evaluaciones, y a su vez las ventajas o potencialidades que trae consigo el hecho de llevar a cabo este tipo de proceso al interior de los sistemas educativos, con el fin de co-ayudar en la búsqueda de consensos que permitan encontrar caminos lógicos y pertinentes sobre la definición de lo que en verdad significa el concepto de evaluación de la calidad educativa.

Del mismo modo, se analizarán algunas implicaciones que tiene la evaluación de la calidad educativa en el desarrollo y fortalecimiento de los sistemas educativos de los países, entendiendo estos como la mejor forma de poder contribuir a que las personas puedan alcanzar un verdadero desarrollo a escala humana, donde al aumentar sus años de escolaridad y poder acceder a sistemas educativos de calidad, les permitan aumentar las posibilidades de mejorar sus condiciones de vida y la de los suyos. De igual forma, se busca evidenciar la situación de la evaluación estandarizada en América Latina, denotando los aspectos en los que ha venido mejorando, pero del mismo modo mostrar cada una de las debilidades que la aplicación de este tipo de evaluaciones trae consigo. Es de anotar que no basta con solo conocer los aspectos antes mencionados, sino que se requiere además conocer los propósitos y el uso que se le da a estos resultados, junto con los criterios necesarios para su divulgación.

Además, la intención será la de realizar un análisis sobre el verdadero desafío que trae consigo el aspecto de la calidad técnica de las ya mencionadas evaluaciones estandarizadas y la importancia de poder constituir unas unidades de evaluación que no solo se encarguen de mostrar resultados para decir si se avanzó o no, sino que además analicen las particularidades de las diversas regiones, con el fin de mostrar análisis contextualizados de las mismas.

Por último, se pretende reflexionar sobre la relación entre los exámenes que se realizan y el concepto de calidad educativa, entendiendo que no debería suponerse, que solo porque una institución educativa no obtiene buenos resultados en una evaluación estandarizada, no significa que no esté realizando buenos ejercicios de calidad educativa, ya que dicho concepto, está cargado por una multiplicidad de elementos que inciden en para que ciertos estudiantes puedan obtener mejores resultados en dichos exámenes, por tanto se hace necesario, no solo denotar dicha relación, sino realizar un análisis a los factores que tienen incidencia en la realización de procesos de calidad educativa. 


\section{Objetivos}

\subsection{Objetivo general}

Analizar la realidad de la evaluación de la calidad educativa en América latina: caso Colombia.

\subsection{Objetivos específicos}

- Identificar los principales conceptos teóricos que tienen relación con el proceso de evaluación de la calidad educativa.

- Conocer el papel de tienen las pruebas estandarizadas en el mejoramiento de la calidad educativa.

- Analizar los procesos evaluativos como instrumentos de diseño de políticas públicas pertinentes.

\section{Evaluación de la calidad educativa}

Antes de poder entender lo que representa el concepto de evaluación de la calidad educativa, es preciso desglosar tal concepto e iniciar por hablar de la importancia de la relación entre evaluación y calidad educativa, la cual es entendida por Egido (2005, p. 15), como "conceptos estrechamente relacionados, hasta el punto de que no podría entenderse la una sin la otra". Lo anterior lleva a entender que los procesos educativos en sí mismos, deben estar permeados en su totalidad por escenarios y actores que propendan por la realización de acciones que permitan contribuir no solo a indicadores entorno a la atención masiva de la población estudiantil, sino que dicha atención que se preste se desarrolle con calidad, tratando de encontrar la denominada pertinencia educativa.

En este sentido es importante mostrar, el aporte realizado por González (2004), el cual afirma que:

Es poco congruente hablar de calidad sin hablar de evaluación, puesto que tildar una cosa como algo que tiene calidad exige realizar una medida, compararla con un referente ideal y elaborar un juicio sobre la adecuación del objeto o sujeto evaluado al referente utilizado. (p. 132)

De acuerdo con lo anterior, es entendible el grado de complejidad que trae consigo el termino evaluación en el campo educativo ya que los elementos de juicio y referentes de lo que cada sociedad considera ideal, complican aún más, la idea de lograr un consenso generalizado sobre la forma como debería ser una verdadera educación de calidad; y esto es muy lógico, si se entiende que cada comunidad posee sus particularidades, su acervo cultural y también sus potencialidades, que hace mucho más complejo el pretender estandarizar dichos procesos evaluativos.

Además de lo planteado anteriormente, es importante ver que el concepto de calidad educativa tiende a concebirse como difuso, debido a la subjetividad de los sujetos, los cuales cada uno tiene una idea distinta de lo que puede llegar a significar, lo que lleva a necesitar urgentemente un consenso que permite mínimamente establecer ciertos lineamientos necesarios que posibilite no solo su entendimiento, sino también su efectiva aplicabilidad.

En este sentido, Cano (1998) sostiene que: 
El concepto de calidad es ante todo subjetivo, ya que cada consumidor o usuario tiene una idea distinta de lo que se entiende por él. Sin embargo, todo el mundo está de acuerdo en que hablamos de la calidad cuando vemos cubiertas todas nuestras expectativas, tanto si se trata de un producto o de un servicio. (p. 60)

La anterior afirmación refuerza el hecho que el fin del concepto de calidad en el campo educativo, deberá ser el poder lograr que los actores de las comunidades educativas puedan lograr un grado de satisfacción tal, que les permita crecer no solo en su aspecto académico, sino también en su crecimiento como persona y por ende que permita el resolver de los diversos problemas que pueden afectar dicho contexto.

No obstante, lo anterior, es relevante el mencionar que el concepto de calidad educativa no es propio del ámbito educativo y que este tuvo sus orígenes en el entorno de los negocios, donde la calidad a la que se referían era la que se veía representada en la producción de diferentes productos, lo cual es algo absurdo cuando se trata de asimilar en el campo educativo, ya que es ilógico tratar de comparar la fabricación de un producto, con la formación de seres humanos. Asimismo, el concepto fue evolucionando en la medida en que los diferentes países fueron logrando ciertos logros a nivel de acceso de la población al servicio educativo, ya que después de lograr que las personas se acercaran a las aulas, el acto seguido estaba orientado a que dicho servicio se lograra prestar no solo en las mejores condiciones, sino también con los mejores estándares de calidad posible. Aquí lo importante en estos momentos, no es el conocer de dónde se originó el concepto, sino entender que los procesos de evaluación de la calidad educativa, no debe ser un proceso unilateral donde los dirigentes toman medidas arbitrarias, sino que sean unas decisiones basadas en los resultados obtenidos, teniendo en cuenta que deben convertirse en decisiones informadas, donde se tome en cuenta las opiniones, particularidades y potencialidades de los diversos actores que intervienen en dicho proceso.

Por otra parte, es relevante saber que, debido a la complejidad del término en mención, existen una serie de elementos que rodean su accionar, como lo son los posibles riesgos que la aplicación del concepto de evaluación de la calidad educativa trae consigo; como lo es la dificultad de lograr que dichas evaluaciones sean objetivas, así también como la pretensión de querer generalizar u homogenizar a las instituciones, cuando lo importante es entender la diversidad de cada institución educativa. De igual forma otro de los riesgos se debe a que muchos de los sistemas evaluativos poseen una estructura prestablecida, que no permiten ningún tipo de cambios o sugerencias, quedándose más en los resultados cuantitativos, y desconociendo en muchas ocasiones, los procesos académicos que se llevan a cabo al interior de las instituciones educativos.

Lo anterior es sustentado por Bolívar (1994) el cual plantea que:

Se ha logrado crear una expectativa infundada, donde la evaluación no es más que un instrumento retórico que sirve para legitimar el funcionamiento actual de los centros, para justificar las reformas o para utilizarse como mecanismo de producción de verdad, en el sentido en que crea realidades. (p. 19)

Pero además se debe comprender que no todo entorno a dichos procesos evaluativos es malo, ya que también existen posturas que defienden las potencialidades de dichos procesos, entre las que sostiene que la financiación de la educación debe realizarse de forma racional con el fin de lograr un mayor grado de equidad, de igual forma se considera que este tipo de evaluaciones aporta información de gran utilidad sobre el estado en el que 
se encuentran los sistemas y que por ende permite diagnosticar la situación en el que este se encuentra.

\section{4. ¿Por qué las pruebas estandarizadas no miden la calidad educativa?}

Es importante el iniciar esta reflexión sobre la pertinencia y efectividad de las pruebas estandarizadas para medir la calidad educativa, partiendo de aclarar algunos interrogantes como, ¿‘uál es el verdadero objetivo de las pruebas estandarizadas?, ¿para qué sirven dichas pruebas?, ¿cómo abordan las pruebas estandarizadas el concepto de calidad educativas?

El poder entender los anteriores interrogantes, ayudará a dilucidar de forma acertada el cuestionamiento inicial, es por esto que se debe iniciar por responder lo que significa una prueba estandarizada, la cual según (Popham, 1999, pp. 2-11) afirma que "una prueba estandarizada es cualquier examen que se administra y califica siguiendo un procedimiento estándar predeterminado. Hay dos tipos principales de pruebas estandarizadas: las pruebas de aptitud y las pruebas de logros".

De acuerdo a lo anterior es claro que las pruebas estandarizadas lo que buscan es realizar una inferencia acertada sobre los conocimientos que poseen los estudiantes en algunas áreas particulares, lo que lleva a pensar que desde su creación este tipo de pruebas no se compromete a evaluar la calidad educativa, ya que su objetivo y alcance se quedan cortos para lograr dicho fin; y esto se entiende desde la perspectiva que dice que un solo instrumento sería incapaz de valorar de forma acertada los procesos de calidad educativa, debido a los múltiples actores, escenarios, contextos y realidades que intervienen en dicho concepto.

De igual forma es de aclarar que las pruebas estandarizadas lo que buscan es realizar inferencias relativas sobre el estado en que se encuentra un estudiante frente a una destreza en particular, lo que posibilita que estos estudiantes se puedan comparar con otros del país. Y es precisamente en el punto anterior donde se presenta la discrepancia, entendiendo que la manera más adecuada de comparar algo, es estableciendo unos ítems de comparación iguales para todos, y como se dijo anteriormente esto sería casi imposible, debido a la multiplicidad de contextos y la gran variedad de formas de enseñar y aprender que poseen los actores del acto educativo.

Además de los elementos anteriores, es de anotar que los docentes se encuentran inmersos en una gran presión por parte de los gobiernos, donde los establecimientos son valorados de acuerdo con los puntajes que obtengan los estudiantes en las pruebas estandarizadas, lo que hace que en muchos de estos establecimientos educativos se preocupen más por preparar a los estudiantes hacia la presentación de dichas pruebas, que de lograr formar buenos seres humanos con calidad. Lo anterior se agrava aún más, cuando empresas externas ofrecen cursos preparatorios que solo buscan instruir a los estudiantes sobre cómo resolver esas pruebas, preocupándose por la instrucción y no por la formación.

En consecuencia con lo anterior existen algunas razones que ayudan a entender que este tipo de pruebas no pueden evaluar la calidad educativa, donde la poca concordancia entre lo que se evalúa y lo que se enseña juegan un papel fundamental para reforzar esta teoría; 
esto se evidencia en la falta de unificación de currículos, los cuales al momento de evaluar los conocimientos a los estudiantes, hace difícil encontrar concordancia entre los contenidos enseñados y los evaluados, y aunque para el caso de Colombia existan unos lineamientos curriculares, donde el Ministerio de Educación Nacional de Colombia (1998) afirma que estos deben orientar la construcción de los planes de estudio, es casi imposible garantizar que las instituciones educativas logren enseñar de forma unificada, debido a que en muchos de los encargados de orientar dicho proceso desconocen el contenido de dichos lineamientos.

Otra de las razones por las cuales estas pruebas no pueden evaluar la calidad educativa, es lo que se denomina causalidad confusa, donde el problema está relacionado con los tres factores que contribuyen al puntaje de los estudiantes en las pruebas estandarizadas de logros; primero lo que se enseña en la escuela, segundo la capacidad intelectual innata del estudiante, y tercero el aprendizaje del estudiante fuera de la escuela; y son precisamente los elementos anteriores los que mayor injerencia tienen al momento de pretender valorar la calidad educativa, porque si los elementos que se evalúan, fueran iguales a los que se enseñan en las escuelas, habría algún grado de correlación en los resultados, pero tal y como se mencionó anteriormente, esto no se cumple, además en este factor tienen que ver no solo lo que se enseña en la escuela, sino también la forma como se enseña en estas, donde la formación y capacitación docente hace que se poseen multiplicidades de metodologías para realizar las respectivas transposiciones didácticas.

Asimismo, otro factor determinante es el relacionado con la capacidad intelectual de los estudiantes, donde es claro mencionar que los niños no nacen con las mismas capacidades, lo que hace incoherente que se pretenda hacer ver como si así fuera; lo más pertinentes es entender la gran diversidad de inteligencias que existen, las cuales no son tenidas en cuenta por las pruebas estandarizadas. Al respecto Gardner (1998) afirma que:

Todos tenemos siete inteligencias modulares, es decir, cada inteligencia es una competencia autónoma e independiente de las otras; se combina de manera adaptativa para el individuo y la cultura, pero no se influyen entre sí. Las inteligencias son: musical, lógico matemática, espacial, lingüistica, cenestésico-corporal, intrapersonal e interpersonal. (p. 51)

Por tanto, las pruebas estandarizadas si pretenden evaluar la calidad educativa, deben asumir un nuevo enfoque que les permita entender que las condiciones intelectuales que poseen los estudiantes son diferentes entre sí e iniciar a concebir el enfoque de inteligencias múltiples, planteado anteriormente.

En consecuencia, el último factor determinante en la causalidad confusa, es el aprendizaje que se da por fuera de la escuela, es aquí donde en el caso de Colombia, existe una diferenciación entre la educación pública de carácter gratuito y la educación privada, donde esta última es la que posee mejores resultados al momento de presentar las pruebas estandarizadas y esto se explica en gran medida por la posibilidad de realización de actividades extracurriculares de manera frecuente, cosa que es difícil en la educación pública, debido a la falta de recursos para poder acceder a esta herramienta didáctica. Siendo así los estudiantes de la educación pública entrarían a competir en desventaja con aquellos estudiantes que pueden gozar no solo de la posibilidad de acceder a un gran número de actividades extracurriculares, sino también a laboratorios bien dotados, instalaciones de gran nivel y recursos ilimitados. 
Por tanto, es inaudito que se crea que una prueba estandarizada podrá medir los procesos de calidad que se llevan a cabo en las instituciones educativas y más aún si se menciona otro elemento diferenciador como lo es el nivel socioeconómico, el cual según cual sea el estrato al que pertenezca el estudiante, va a tener más o menos posibilidades de acceso a herramientas y materiales de calidad que ayudarían a tener un mejor o peor proceso educativo de calidad.

\section{Complejidad de la medición y evaluación de sistemas educativos}

Es importante iniciar este análisis, conociendo que es a lo que se denomina una evaluación estandarizada, y es aquí donde (Ravela, 2008) afirma que:

Se entiende por evaluación estandarizada en gran escala a aquella que permite producir información comparable acerca de los desempeños de estudiantes pertenecientes a distintos contextos culturales y regionales e, incluso, a distintos países, y que ofrece un panorama de la situación de un país. (pp. 45-63)

En consecuencia, es entendible que la definición anterior permite aclarar que este tipo de evaluaciones tiene su razón de ser en la cantidad de personas en que se pueda aplicar, para poder obtener cierto tipo de información que permita comparar los resultados de diferentes regiones. Además, es de anotar que estas evaluaciones se enfocan en determinar el cambio que han sufrido ciertos aprendizajes y capacidades que demuestran los estudiantes a lo largo de un año lectivo determinado y a su vez la acumulación de conocimientos que estos puedan adquirir a lo largo de su vida estudiantil.

Asimismo, se puede mencionar que a pesar de las concepciones y críticas que poseen este tipo de evaluaciones, también se trata de poner de manifiesto algunas razones por las cuales es importante que estas se realicen, como una forma de conocer la dinámica de los procesos y los resultados de los sistemas educativo; por tanto las evaluaciones estandarizadas pueden servir como base para diseñar o implementar alguna política educativa y a su vez como un instrumento para conocer el estado de los aprendizajes de los estudiantes en ciertos campos del saber. En este sentido se puede mencionar que la mayoría de este tipo de evaluaciones tal y como están en algunos países, solo ayuda a visualizar los resultados educativos de un conjunto de estudiantes y a entregar información sobre algunas capacidades que alcancen los estudiantes.

No cabe duda entonces, que las evaluaciones estandarizadas bien diseñadas y bien ejecutadas, podrían ser una fuente valiosa tanto de información, como de insumo para que los gobiernos puedan establecer políticas educativas pertinentes y contextualizadas, pero se debe entender que dichas evaluaciones solo ofrecen un indicador parcial dentro de todo el universo llamado calidad educativa, a su vez entender que estas evaluaciones solo son un componente de un sistema de evaluación integra del campo educativo, por tanto hace que estas sean necesarias, pero no suficientes.

Por otra parte, es indudable que si se toca la situación de la evaluación estandarizada en América Latina, se debe mencionar que la participación de los países de la región ha venido aumentando tanto en estudios organizados por la OREAL/UNESCO, como en sistemas de evaluación organizados por el Laboratorio Latinoamericano de Evaluación de la Calidad de la Educación (LLECE) y en la participación en pruebas internacionales como 
las pruebas PISA, a su vez la destacada participación de algunos países en el constituido grupo iberoamericano de PISA, lo cual es relevante, si se pretende entender en contexto los resultados de dichas pruebas; por tanto, se puede mencionar que estas evaluaciones estandarizadas en algunos aspectos están teniendo avances, como por ejemplo en la transparencia y creciente atención en la difusión y uso de los resultados, de igual forma se está trabajando para que cada vez más se pueda pasar de las actuales pruebas normativas a unas pruebas de criterios que se enfoquen en que es lo que verdaderamente el estudiante sabe y es capaz de hacer; asimismo, se ha venido aumentando la preocupación por iniciar investigaciones que se enfoquen en cada vez más factores que tengan incidencia en el proceso de aprendizaje de los estudiantes, como una manera de avanzar en la constitución de un sistema de evaluación cada vez más integral.

No obstante, a lo anterior, estas evaluaciones poseen una serie de debilidades que es necesario que se analicen, como por ejemplo afirma Ferrer (2006), la confusión que suscita el no saber a ciencia cierta la funcionalidad y propósitos de cada tipo de evaluación, como elemento fundamental para poder poseer un acertado sistema evaluativo, por otra parte se necesita que haya una mayor discusión pública sobre la concurrencia entre lo que se evalúa y lo que se enseña, en pocas palabras se necesita que se puedan mejorar las investigaciones sobre las explicaciones del porque se obtienen ciertos resultados, y no solo eso, sino que a su vez se puedan implementar políticas de estado que posibiliten resolver la problemática identificada con dichas evaluaciones.

En concordancia con lo anterior, Murillo y Román (2010) sostienen que:

El primer paso que deben asumir los gobiernos, debe ser el de definir cuál debe ser el propósito de su sistema de evaluación y para que se usaran los resultados de las mismas, y que es necesario que se establezca un adecuado equilibrio entre la implementación de pruebas nacionales y la participación en las pruebas internacionales. (pp. 97-120)

Por tanto, se requiere de un sistema de evaluación que desarrolle exámenes de alta calidad técnica, permeado por principios de transparencia y responsabilidad con los resultados, donde se puedan crear y fortalecer unas unidades de evaluación dotadas de elementos técnicos y de calidad, donde su independencia para analizar y publicar resultados sea su principio fundamental y que además se les brinde los presupuestos necesarios para poder operar de la mejor forma.

\section{La evaluación como instrumento de política educativa}

Para iniciar con el análisis de la evaluación como instrumento de política pública, es importante entender que el enfoque evaluativo abordado es el concerniente a un tipo de evaluación de competencias, que es el que utilizan las instituciones globalizadoras como la OCDE, y que por ende entran a hacer parte de todo tipo de reformas en las agendas de los diferentes países. No cabe duda, que pareciera que nos encontráramos en un proceso expansionista o colonialista en el ámbito educativo, debido a que las agendas de políticas educativas están más influenciadas por las directrices de organismos internacionales que, por las verdaderas necesidades del contexto, ya que son estas las que realizan ciertas exigencias educativas, articuladas a temas económicos.

Lo anterior, nos lleva a analizar cuidadosamente sobre la necesidad de entender los factores inherentes a la obtención no solo de resultados en evaluaciones estandarizadas, 
sino también en los procesos de calidad educativa, al respecto Taba (1993) afirma que la evaluación es un proceso mediante el cual se obtiene información que permite determinar si un determinado programa educativo ha arrojado algún efecto; por tanto se puede decir que la realización de este tipo de evaluaciones es necesaria a fin de obtener información constante y real de algunos aspectos que constituyen la calidad educativa, pero que no se debe supeditar solo al aspecto academicista de los resultados de estas pruebas.

Ahora bien, se debe abordar este tema desde la realidad circundante, como lo es el caso de Colombia, donde el tema evaluativo es asumido por el Instituto Colombiano de Fomento de la Educación Superior (ICFES), el cual desde 1968, ha venido encargándose de estos temas. Desde el año 2002, el Ministerio de Educación Nacional de Colombia (MEN) comenzó a cambiar el enfoque de las evaluaciones nacionales, al pasar de evaluar conocimientos a la evaluación de competencias, el cual asume que "las competencias hacen referencia a los conocimientos y habilidades, así como a las estrategias y rutinas necesarias para aplicarlos, que junto con las emociones y actitudes apropiadas permiten atender demandas y tareas a cabalidad" (Simone y Hersh, 2000, p. 4).

En la actualidad el ICFES, es el encargado de realizar las denominadas pruebas saber, que se realizan en los grados tercero y quinto de primaria, y a su vez en el grado noveno de secundaria, y once de la media vocacional, estas se realizan con el fin de realizar un muestreo del avance que pueden ir teniendo los estudiantes a lo largo de su vida estudiantil y aunque en la actualidad estas pruebas han mejorado considerablemente en la estructura de las preguntas, se hace necesario, la inclusión de elementos contextuales para que los resultados sean acordes a las condiciones sociales de los estudiantes. Asimismo, se realizan las pruebas ECAES con el fin de evaluar los aprendizajes y competencias de los estudiantes que concluyen el ciclo de educación superior.

De acuerdo con lo anterior, se evidencia que el organismo encargado del sistema evaluativo del país adopta un concepto de competencia que difiere con el utilizado por los organismos internacionales como la OCDE (2005), el cual plantea que, una "competencia es algo más que únicamente conocimientos y habilidades. Implica la habilidad para enfrentarse a demandas complejas, haciendo uso y movilizando recursos psicosociales (que incluyen habilidades y actitudes) en un contexto particular". Lo que se plantea es poder utilizar un concepto un poco más completo de lo que es evaluar por competencias donde no solo se base en conocimiento y habilidades, sino que aborde otros elementos complementarios. En este sentido Vasco (2003), afirma que:

Conjunto de conocimientos, habilidades, actitudes, comprensiones y disposiciones cognitivas, metacognitivas, socioafectivas, comunicativas y psicomotoras apropiadamente relacionadas entre sí para facilitar el desempeño flexible, eficaz y con sentido de una actividad o de cierto tipo de tareas en contextos relativamente nuevos y retadores. (pp. 10-14)

Lo anterior, lleva a pensar que el sustento teórico que orienta los sistemas evaluativos de un país debería enriquecerse de otros autores que poseen una visión más integral del tema, para así poder buscar una articulación, entre lo que es enseñar por competencias y evaluar por competencias, porque de lo contrario, siempre existirá la disparidad, entre lo que se enseña y lo que se evalúa.

Sin embargo, la preocupación radica en que, si se utilizan solo las pruebas estandarizadas para medir la calidad educativa, hace que se pierda un poco los procesos de evaluación formativa en las aulas, desconociendo la importancia de esta en la formación de ambiente 
educativos y sobre todo con los estudiantes que poseen desempeños bajos. Si se quiere avanzar hacia el enfoque de calidad educativa, se debe generar una reflexión crítica y académica de las políticas educativas, donde puedan intervenir todos los actores educativos, y juntos poder construir un consenso sobre lo que significa calidad educativa a partir de las realidades sociales y culturales de cada contexto. En este sentido, Murcia (1991) plantea que:

La acción evaluativa supone momentos de reflexión crítica, sobre el estado de un proceso del
que se tiene una información sistemáticamente recopilada, a la luz de unos principios y propó-
sitos previamente definidos, con el fin de valorar esa información y tomar decisiones
encaminadas a reorientar el proceso; es por eso que para nosotros la acción de evaluación se
constituye en un elemento dinamizador fundamental para el estudiante, el maestro, la escuela,
la familia y la comunidad. (p. 79)

\section{Aspectos metodológicos}

Es de anotar, que el alcance del presente estudio fue de tipo descriptivo, debido a que se buscó enunciar de forma ordenada y coherente los elementes que fundamentan el proceso de evaluación de la calidad educativa en Colombia, por tanto, se entiende que el enfoque utilizado fue un enfoque cualitativo, orientado por un modelo epistemológico histórico hermenéutico que busca interpretar y comprender los motivos internos de la acción humana, mediante procesos libres, no estructurados, sino sistematizados, que tienen su fuente en la filosofía humanista.

Asimismo, se utilizaron técnicas de análisis documental entendidas estas como un conjunto de operaciones destinadas a representar el contenido y la forma de un documento para facilitar su consulta o recuperación, o incluso para generar la consolidación de conceptos, donde los instrumentos como las fichas bibliográficas y los cuadros de análisis documental fueron relevantes para poder analizar la realidad de los procesos de evaluación de la calidad educativa en Colombia. En ese orden de ideas, se analizaron documentos e informes, a fin de poder obtener no solo datos confiables, sino también información documental relevante que contribuyera a alcanzar el objetivo propuesto.

Los anteriores instrumentos investigativos, ayudaron no solo a detectar, sino a su vez a seleccionar y clasificar los elementos fundamentales de cada texto a partir de procesos de codificación que ayudaron a establecer las categorías principales del presente estudio, entendiendo que dicho proceso hermenéutico seria esencial para entender no solo las variables que tenían relación con el tema en mención, sino también que posibilitaría entender las relaciones que se entretejen entre ellos.

\section{Conclusiones}

Después de conocer las diversas posturas que rodean el concepto de evaluación de la calidad educativa, se llega a la conclusión de que actualmente es difícil llegar a consensos que permitan unificar criterios sobre su correcta aplicabilidad, en lo que si se está de acuerdo es en la necesidad de que los procesos se puedan evaluar con el fin de corregir los elementos que no estén funcionando de la mejor manera y fortalecer los elementos que estén dando resultados. 
Otro elemento importante es que para lograr una debida aplicación se deben considerar todas las singularidades de los sistemas educativos, no con el fin de homogenizar, sino por el contrario, de reconocer particularidades de los diversos contextos, los procesos de evaluación en el campo educativo deberán poseer un alto grado de consenso, donde la participación de los actores que intervienen sea muy activa. Lo anterior lo mencionan Harvey y Green (1993) cuando afirman que en una sociedad democrática debe existir espacio para personas que sostengan diferentes visiones. Se puede decir que evidentemente las pruebas estandarizadas no están diseñadas para evaluar la calidad educativa, pero si poseen facilidad para establecer un dominio relativo de contenidos que logran los estudiantes, y se dice relativo ya que estas pruebas no tienen en cuenta los ítems de mayor o menor contestación, sino los intermedios.

De igual forma se puede decir con certeza que si se pretende evaluar la calidad educativa se deben tener en cuenta una serie de factores determinantes, como lo es encontrar concordancia entre lo que se enseña y se evalúa, el entender que los niños no nacen con las mismas capacidades, lo que hace imposible que un instrumento de evaluación se pueda aplicar de la misma forma y pretendiendo obtener los mismos resultados en todos.

Asimismo los maestros deben estudiar y entender la forma como se diseñan y se llevan a cabo las pruebas estandarizadas, llegando a dilucidar que se pregunta y con qué fin se pregunta a sus estudiantes, lo que ayudará a bajar la presión de categorizar erradamente a las escuelas de acuerdo a dichos puntajes obtenidos, explicando a los demás actores que dichos resultados suministran una mirada engañosa de calidad educativa y poder aportar otra serie de evidencias que muestre que el trabajo de educar no se puede medir con una simple prueba estandarizada, que asume que todos los niños son iguales y que viven y poseen las mismas condiciones socioeconómicas.

El análisis de la situación de las evaluaciones estandarizadas en América Latina trae consigo algunas reflexiones finales entorno a la necesidad de entender que la implementación de este tipo de evaluaciones solo tendrá efectos positivos, si se conciben y emplean como un insumo esencial para la toma de decisiones que lleven al diseño de política pública educativa que se encargue de resolver los problemas que se logren detectar en los resultados que se obtengan, ya que si estos resultados no se conocen, ni se utilizan para dicho fin, no tendrían mayor relevancia, ya que no tiene ningún sentido el solo mostrar resultados como una forma de rendir cuentas, sin realizar un análisis contextual e integral de estas evaluaciones.

De igual forma se requiere de la implementación de instrumentos complementarios que contribuyan a tener una visión integral de los resultados, donde no solo importe si el estudiante aprobó o no, sino que también se preocupen por establecer particularidades culturales y de contextos que son relevantes al momento de implementar sistemas evaluativos pertinentes y de calidad, que posibiliten el utilizar dichos resultados para diseñar políticas públicas que ayude a resolver los problemas que se identifiquen con estas pruebas. Además, los gobiernos se deben comprometer en realizar de forma responsable una divulgación de resultados con todos los actores del proceso educativo, para lograr una mayor retroalimentación del proceso, en pocas palabras es necesario que estas evaluaciones se puedan combinar con estudios cualitativos para lograr una mayor integralidad en dichas pruebas y donde la unidades locales de evaluación posea los recursos técnicos y financieros, ya que evaluar bien, requiere de la mejor y mayor inversión 
posible. Es de resaltar que Colombia ha realizado esfuerzos, para avanzar en la consolidación de un sistema de evaluación que permita mejorar en las pruebas estandarizadas, tanto internas como externas, por tal razón ha venido diseñando y poniendo en marcha algunas políticas educativas que, aunque son un avance, siguen siendo insuficientes.

Asimismo, tanto las pruebas nacionales como las internacionales permiten ver el bajo nivel de competencias que tienen los estudiantes colombianos en matemáticas. Según el ICFES (2013), el 44\% de los estudiantes colombianos finaliza la educación media sin desarrollar las competencias básicas en esta materia, mientras que según PISA este indicador llega al $70 \%$. Por tal razón, en el año 2015 se creó el Índice Sintético de la Calidad educativa, para medir el progreso en las pruebas SABER de un año a otro, la eficiencia o tasas de repitencia, el desempeño que consiste en el puntaje de las pruebas SABER y el ambiente escolar de todas las instituciones educativas del país. Además, ha iniciado con la implementación de la jornada única en los colegios oficiales en Colombia, la cual pretende aumentar un número de horas que permanecen los estudiantes en los planteles educativos, a fin de fortalecer competencias en lenguaje y matemáticas, tendientes a mejorar los resultados en las pruebas estandarizadas.

Es de anotar que las pruebas ya mencionadas pueden tener diferentes funcionalidades, como la posibilidad que le brinda a las familias el poder elegir a las instituciones que posean mejores resultados, de igual forma, pueden servir para la rendición de cuentas, así como también, para realizar pruebas diagnósticas al interior de los establecimientos educativos y en general para aportar al sistema de información general del funcionamiento educativo y a la investigación educativa.

\section{Referencias}

Baena, G. (1998). Técnicas de investigación. Ciudad de México: Editores Mexicanos Unidos.

Bolívar, A. (1994). La evaluación de centros: entre el control administrativo y la mejora interna. En J. M. Escudero Muñoz y M. T. González González (Eds.), Profesores y escuela ¿ihacia una reconversion (pp. 251-282). Madrid: Ediciones Pedagógicas.

Cano, E. (1998). Evaluación de la calidad educativa. Madrid: La Muralla.

CONEVAL. (2012). Informe de pobreza y evaluación en el estado de Sonora. Sonora: Coneval.

CONEVAL. (2012). Porcentaje de población en situación de probreza extrema segun entidad federativa. Sonora: Coneval.

Delval, J. (1992). Aprender a aprender. I. El desarrollo de la capacidad de pensar. Madrid: Alhambra Longman.

Egido, I. (2005). Reflexiones en torno a la evaluación de la calidad educativa. Tendencias Pedagógicas, 10, 17-28.

Ferrer, G. (2006). Sistemas de Evaluación de aprendizajes en América Latina: Balance y Desafios. Lima: PREAL.

Gardner, H. (1998). Inteligencias múltiples. Barcelona: Paidós.

González, A. (2004). Evaluación del clima escolar como factor de calidad. Madrid: La Muralla. 
Harvey, L. y Green, D. (1993). Defining quality. Assessment E evaluation in higher education. Birmingham: QHE.

ICFES. (2013). Sistema Nacional de Evaluación Estándarizada de la Educación: alineación del examen $S A B E R 11^{\circ}$. Bogotá: ICFES.

INEGI. (2013). www.inegi.org.mx. Recuperado de http://www.inegi.org. $\mathrm{mx} /$ sistemas/olap/proyectos/bd/consulta.asp?p=17118\&c=27769 $\& \mathrm{~s}=\mathrm{est}$

Ministerio de Educación Nacional de Colombia. (1998). Lineamientos Curriculares. Bogotá: Magisterio.

Murcia, J. (1991). Proceso pedagógico y evaluación. Madrid: Antropos.

Murillo, F. J. y Román, M. (2010). Retos en la evaluación de la calidad de la educación en América Latina. Revista Iberoamericana de Educación, 53, 97-120.

OCDE. (2005). The definition and selection of key competencies. París: OCDE.

Popham, W. J. (1999). ¿Por qué las pruebas estandarizadas no miden la calidad educativa? Educational Leadership, 56(6), 2-11.

Ramos, R. Y. (2001). Educacion integral. Una educación holística para el siglo XXI. París: Desclée de Brouwer, S.A.

Ravela, P. (2008). Las evaluaciones educativas que América Latina necesita. Revista Iberoamericana de Evaluación Educativa, 1(1), 45-63.

Simone, D. y Hersh, L. (2000). Definition and selection of key competencies. The ines compendium. Recuperado de

https://www.google.es/url? sa=t\&rct=j\&q=\&esrc=s\&source=web\&cd=2\&cad=rja\&uact $=$ 8\&ved=OahUKEwiovOzXgbnYAhUH 1 xQKHU8fDdUQFggwMAE\&url=https\%3A\%2F \%2Fwww.oecd.org\%2Fpisa\%2F35070367.pdf\&usg=AOvVawOcboijnZiyrDTvn 15xdTes

Taba, H. (1993). Elaboración del curriculum. Buenos Aires: Troquel.

Vasco, C. (2003). Estándares básicos de calidad para la educación. Bogotá: Mimeo.

\section{Breve CV del autor}

\section{Deivis Robinson Mosquera Albornoz}

Soy docente nacido en la Ciudad de Istmina, Departamento del Chocó, de nacionalidad colombiano, he realizado la escuela completa para ser maestro, desde la normal superior, pasando por la licenciatura en educación básica y posteriormente la maestría en educación, que han realizado los aportes necesarios para desempeñarme como maestro en una escuela primaria y en la facultad de ciencias de la educación en la Universidad Católica de Oriente en la Ciudad de Rionegro, Antioquia, donde resido, en la actualidad estoy llevando a cabo mis estudios de doctorado en educación, orientando la tesis en la línea de investigación sobre el fortalecimiento de las procesos investigativos en el aula. ORCID ID: O000-00034472-2 104. Email: deromoal@gmail.com 